\title{
Structure of the McMullen domain in the parameter planes for rational maps
}

\author{
by
}

\section{Robert L. Devaney (Boston)}

\begin{abstract}
We show that, for the family of functions $F_{\lambda}(z)=z^{n}+\lambda / z^{n}$ where $n \geq 3$ and $\lambda \in \mathbb{C}$, there is a unique McMullen domain in parameter space. A McMullen domain is a region where the Julia set of $F_{\lambda}$ is homeomorphic to a Cantor set of circles. We also prove that this McMullen domain is a simply connected region in the plane that is bounded by a simple closed curve.
\end{abstract}

Our goal in this paper is to describe some of the dynamical behavior arising in the families of rational maps given by

$$
F_{\lambda}(z)=z^{n}+\lambda / z^{n}
$$

where $\lambda \neq 0$ is a complex parameter and $n$ is a positive integer. In particular, we investigate the structure of a region in the parameter plane that we call the McMullen domain. Parameters drawn from this region have the property that the Julia sets of the corresponding functions are Cantor sets of simple closed curves.

Each of the functions $F_{\lambda}$ has a total of $2 n$ "free" critical points. However, like the well studied quadratic family $Q_{c}(z)=z^{2}+c$, each function really has only one free critical orbit since all forward orbits of the critical points behave symmetrically. Hence the $\lambda$-plane is a natural parameter plane for these families.

As another similarity with the quadratic family, the point at $\infty$ is a superattracting fixed point for each $\lambda$ when $n>1$, and so it may be the case that the critical orbits enter the basin of this fixed point. Unlike the quadratic family, however, there are three distinctly different manners in which the critical orbits may escape to $\infty$, and this in turn determines the topological structure of the Julia sets for the escape parameters. We denote the immediate basin of attraction of $\infty$ by $B_{\lambda}$. One possible escape scenario is that the critical values all lie in $B_{\lambda}$. Since 0 is a pole, there is a

2000 Mathematics Subject Classification: 37F10, 37F45. 
neighborhood of 0 that is mapped into $B_{\lambda}$. Now either this neighborhood is itself contained in $B_{\lambda}$, or else 0 lies in a disjoint preimage of $B_{\lambda}$, in which case we denote this preimage by $T_{\lambda}$. In the latter case, note that $F_{\lambda}$ maps $T_{\lambda}$ in $n$-to-one fashion onto $B_{\lambda}$ while $F_{\lambda} \mid B_{\lambda}$ is also $n$-to-one. Hence the only preimages of $B_{\lambda}$ are $B_{\lambda}$ itself and $T_{\lambda}$. So a second possible scenario occurs when the critical values lie in $T_{\lambda}$ rather than $B_{\lambda}$. Finally, the third possibility arises when some higher iterate of the critical values lies in $T_{\lambda}$. The following theorem describes the Julia sets that result from these three different situations [7]:

Theorem (The Escape Trichotomy). For the family of functions

$$
F_{\lambda}(z)=z^{n}+\lambda / z^{n}
$$

with $n \geq 2$ and $\lambda \in \mathbb{C}$ :

(i) If the critical values lie in $B_{\lambda}$, then the Julia set is a Cantor set.

(ii) If the critical values lie in $T_{\lambda}$, then the Julia set is a Cantor set of simple closed curves.

(iii) If the critical values lie in any other preimage of $T_{\lambda}$, then the Julia set is a Sierpiński curve.

A Sierpiński curve is a planar set that is characterized by the following five properties: it is a compact, connected, locally connected and nowhere dense set whose complementary domains are bounded by simple closed curves that are pairwise disjoint. It is known from work of Whyburn [19] that any two Sierpiński curves are homeomorphic. In fact, they are homeomorphic to the well known Sierpiński carpet fractal. From the point of view of topology, a Sierpiński curve is a universal set in the sense that it contains a homeomorphic copy of any planar, compact, connected, one-dimensional set [15]. The first example of a Sierpiński curve Julia set was given by Milnor and Tan Lei [14]. See also Ushiki [18].

Case (ii) of the Escape Trichotomy was first observed by McMullen [11], who showed that this phenomenon occurs in each family provided that $n \neq$ 1,2 and $|\lambda|$ is sufficiently small.

In Figures 1 and 2 we display a Julia set drawn from the family $F_{\lambda}(z)=$ $z^{3}+\lambda / z^{3}$ corresponding to each of the three cases in the Escape Trichotomy.

In Figure 3 we display the parameter plane for the family $F_{\lambda}(z)=z^{3}+$ $\lambda / z^{3}$. The black regions indicate parameter values for which the critical orbit does not escape to $\infty$. Again in analogy with the quadratic polynomial family, for these parameters the Julia set is a connected set. The colored regions in this picture represent $\lambda$-values for which the critical orbit tends to $\infty$. The exterior region corresponds to parameter values for which the Julia set is a Cantor set; we call this set the Cantor set locus. The small region in the center corresponds to parameter values for which the Julia set 


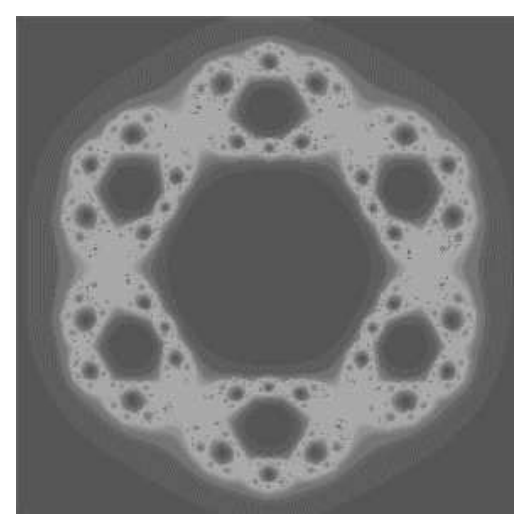

(a)

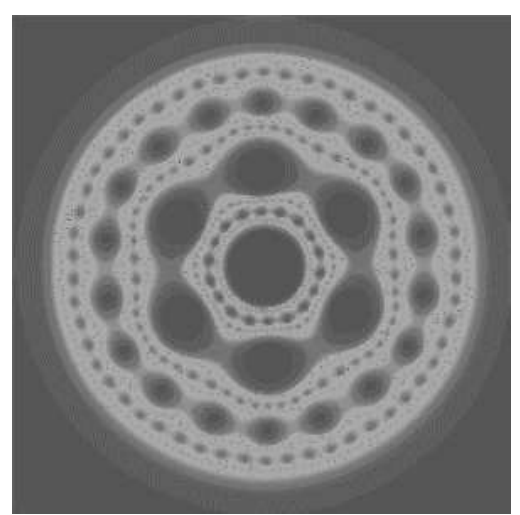

(b)

Fig. 1. Several Julia sets from the the family $z^{3}+\lambda / z^{3}$. The Julia set in Figure (a) is a Cantor set; Figure (b) is a Cantor set of simple closed curves.

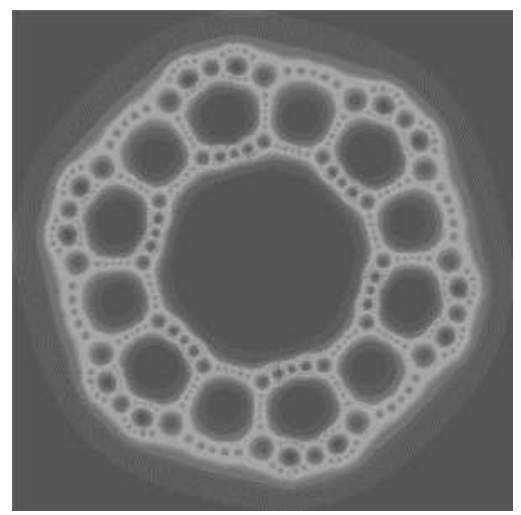

Fig. 2. Another Julia set from the family $z^{3}+\lambda / z^{3}$; this Julia set is a Sierpiński curve.

is a Cantor set of simple closed curves. We call this region the McMullen domain. The other colored regions correspond to parameters for which the Julia set is a Sierpiński curve. These are called Sierpiński holes.

It is known [1], [5] that there are infinitely many disjoint Sierpiński holes for each of these families for each $n>1$. Roesch [17] has shown that each Sierpiński hole is homeomorphic to a disk when $n=2$.

In Figure 4 we have displayed the parameter plane in the case $n=4$. The McMullen domain is again the centrally located colored region in this plane, and all of the other such regions that are bounded are Sierpiński holes.

Note that, in the case $n=3$, there appear to be two large copies of a Mandelbrot set that straddle the positive and negative real axes, and when $n=4$, there are three symmetrically-located copies of Mandelbrot 


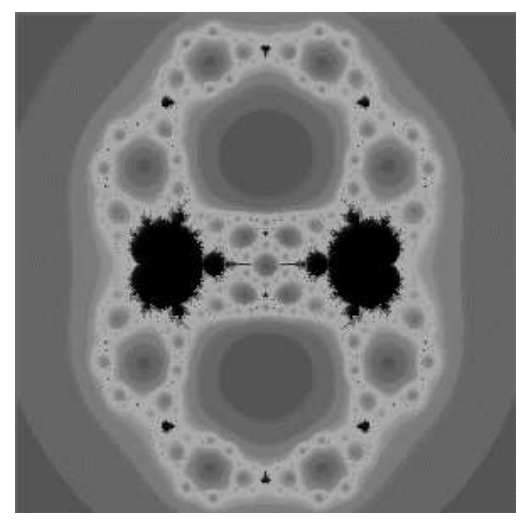

Fig. 3. The parameter plane for the family $z^{3}+\lambda / z^{3}$.
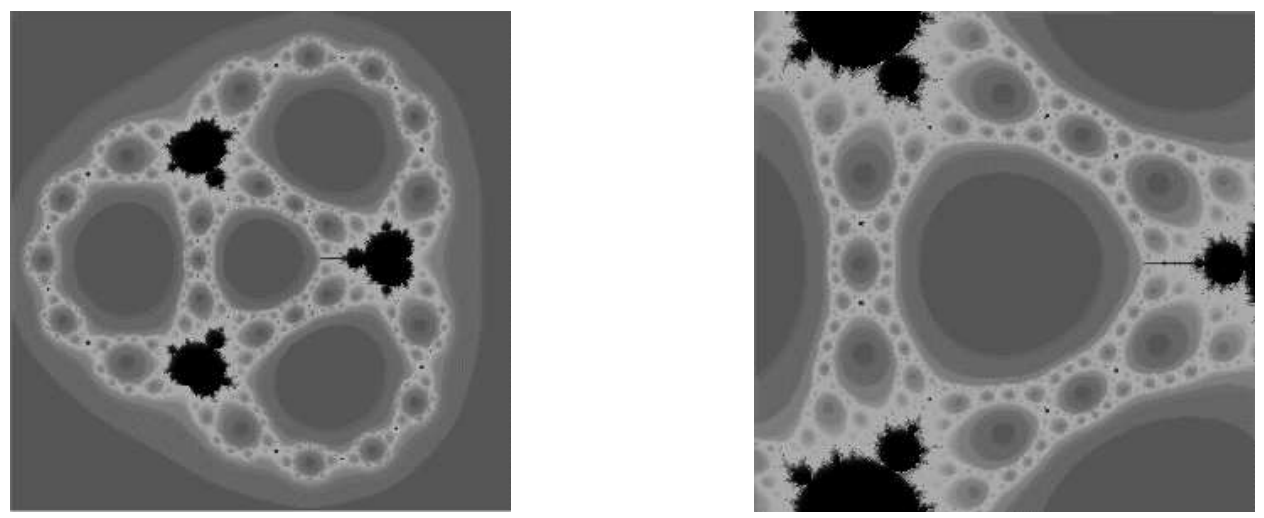

Fig. 4. The parameter plane for the family $z^{4}+\lambda / z^{4}$ and a magnification around the McMullen domain.

sets. These are called the principal Mandelbrot sets for $F_{\lambda}$. By the DouadyHubbard theory of polynomial-like maps, it is known that there are $n-1$ such sets in the parameter plane for $z^{n}+\lambda / z^{n}$ and that these sets are homeomorphic to the standard quadratic Mandelbrot set [3]. Moreover, for parameters drawn from these sets, there is an invariant subset on which $F_{\lambda}$ is conjugate to the corresponding quadratic polynomial on its Julia set.

Our goal in this paper is to investigate further properties of the parameter plane for these maps and, in particular, the structure of the McMullen domain. Our main goal is to prove:

THEOREM. There is a unique McMullen domain in the parameter plane for each $n \geq 3$. Moreover, this region is an open disk that is bounded by a simple closed curve. 
We observe that it is rare in complex dynamics for an "escape" region to be bounded by a nice simple closed curve. For example, the boundary of the escape locus for quadratic polynomials is the boundary of the Mandelbrot set, which is far from being a simple closed curve.

1. Elementary mapping properties. In this paper we restrict attention to the family of rational maps given by

$$
F_{\lambda}(z)=z^{n}+\lambda / z^{n}
$$

where, in view of McMullen's result, we assume that $n \geq 3$.

In the dynamical plane, the object of principal interest is the Julia set of $F_{\lambda}$, which we denote by $J\left(F_{\lambda}\right)$. The Julia set is the set of points at which the family of iterates $\left\{F_{\lambda}^{n}\right\}$ fails to be a normal family in the sense of Montel. It is known that $J\left(F_{\lambda}\right)$ is also the closure of the set of repelling periodic points for $F_{\lambda}$ as well as the boundary of the set of points whose orbits escape to $\infty$ under iteration of $F_{\lambda}$. See [13].

The point at $\infty$ is a superattracting fixed point for $F_{\lambda}$ and we denote the immediate basin of $\infty$ by $B_{\lambda}$. It is well known that $F_{\lambda}$ is conjugate to $z \mapsto z^{n}$ in a neighborhood of $\infty$ in $B_{\lambda}$ (see [16]). There is also a pole of order $n$ for $F_{\lambda}$ at the origin, so there is a neighborhood of 0 that is mapped into $B_{\lambda}$ by $F_{\lambda}$. If this neighborhood is disjoint from $B_{\lambda}$, then we denote the preimage of $B_{\lambda}$ that contains 0 by $T_{\lambda}$. So $F_{\lambda}$ maps both $B_{\lambda}$ and $T_{\lambda}$ in $n$-to-one fashion over $B_{\lambda}$. We call $T_{\lambda}$ the trap door since any orbit that eventually enters the immediate basin of $\infty$ must "fall through" $T_{\lambda}$ en route to $B_{\lambda}$.

The map $F_{\lambda}$ has $2 n$ other critical points given by $\lambda^{1 / 2 n}$. We call these points the free critical points for $F_{\lambda}$. There are, however, only two critical values, and these are given by $\pm 2 \sqrt{\lambda}$. We denote a free critical point by $c_{\lambda}$ and a critical value by $v_{\lambda}$. The map also has $2 n$ prepoles given by $(-\lambda)^{1 / 2 n}$. Note that all of the critical points and prepoles lie on the circle of radius $|\lambda|^{1 / 2 n}$ centered at the origin. We call this circle the critical circle and denote it by $C_{\lambda}$.

The map $F_{\lambda}$ has some very special properties when restricted to circles centered at the origin:

Proposition.

(i) $F_{\lambda}$ takes the critical circle $2 n$-to-one onto the straight line connecting the two critical values $\pm 2 \sqrt{\lambda}$.

(ii) $F_{\lambda}$ takes any other circle centered at the origin to an ellipse whose foci are the critical values.

Proof. For (i), let $\lambda=|\lambda| \exp (i \mu)$. We compute

$$
\begin{aligned}
F_{\lambda}\left(|\lambda|^{1 / 2 n} e^{i \theta}\right) & =|\lambda|^{1 / 2}((\cos n \theta+\cos (\mu-n \theta))+i(\sin n \theta+\sin (\mu-n \theta))) \\
& =|\lambda|^{1 / 2}(x+i y) .
\end{aligned}
$$


A straightforward computation then shows that

$$
\frac{d}{d \theta}\left(\frac{y}{x}\right)=0
$$

so that the image of the critical circle has constant slope and so is a line segment. One checks easily that the extremities of this segment occur at $\pm 2 \sqrt{\lambda}$.

For (ii), another computation shows that, on the circle of radius $r$ centered at the origin, we have

$$
\left|F_{\lambda}\left(r e^{i \theta}\right)+2 \sqrt{\lambda}\right|+\left|F_{\lambda}\left(r e^{i \theta}\right)-2 \sqrt{\lambda}\right|=2\left(r^{n}+|\lambda| / r^{n}\right),
$$

which is constant. So the image of this circle is an ellipse with foci at the critical values $\pm 2 \sqrt{\lambda}$.

We call the image of the critical circle the critical segment. We call the straight ray connecting the origin to $\infty$ and passing through one of the critical points (resp., prepoles) a critical point ray (resp., prepole ray). Similar straightforward computations show that each of the critical point rays is mapped in two-to-one fashion onto one of the two straight line segments of the form $t v_{\lambda}$, where $t \geq 1$ and $v_{\lambda}$ is the image of the critical point on this ray. So the image of a critical point ray is one of two straight rays connecting $\pm v_{\lambda}$ to $\infty$. Hence the critical segment together with these two rays forms a straight line through the origin.

Similarly, each of the $2 n$ prepole rays is mapped in one-to-one fashion onto the same straight line given by $i t \sqrt{\lambda}$, where $t$ is now any real number. Note that the image of the prepole rays is the line that is perpendicular to the line $t v_{\lambda}$ for $t \in \mathbb{R}$ which contains the critical segment and the images of the critical point rays.

Let $\mathcal{S}_{\lambda}$ be an open sector bounded by two prepole rays corresponding to adjacent prepoles on $C_{\lambda}$, i.e., $\mathcal{S}_{\lambda}$ is a sector in the plane with angle $2 \pi / 2 n$. We call $\mathcal{S}_{\lambda}$ a critical point sector since it contains at its "center" a unique critical point of $F_{\lambda}$. Similarly, let $\mathcal{P}_{\lambda}$ be the open sector bounded by two critical point rays corresponding to adjacent critical points on $C_{\lambda}$. We call $\mathcal{P}_{\lambda}$ a prepole sector. The next result follows immediately from the above:

Proposition (Mapping properties of $F_{\lambda}$ ).

(i) $F_{\lambda}$ maps each critical point sector in two-to-one fashion onto the open half-plane that is bounded by the image of the prepole rays and contains the critical value that is the image of the unique critical point in the sector.

(ii) $F_{\lambda}$ maps each prepole sector in one-to-one fashion onto the entire plane minus the two half-lines $\pm t v_{\lambda}$ where $t \geq 1$. 
(iii) $F_{\lambda}$ maps the region in both the interior and the exterior of the critical circle onto the complement of the critical segment as an n-to-one covering map.

We now turn to the symmetry properties of $F_{\lambda}$ in both the dynamical and parameter planes. Let $\nu$ be the primitive $(2 n)$ th root of unity given by $\exp (\pi i / n)$. Then, for each $j$, we have $F_{\lambda}\left(\nu^{j} z\right)=(-1)^{j} F_{\lambda}(z)$. Hence, if $n$ is even, we have $F_{\lambda}^{2}\left(\nu^{j} z\right)=F_{\lambda}(z)$. Therefore the points $z$ and $\nu^{j} z$ land on the same orbit after two iterations and so have the same eventual behavior for each $j$. If $n$ is odd, the orbits of $F_{\lambda}(z)$ and $F_{\lambda}\left(\nu^{j} z\right)$ are either the same or else they are the negatives of each other. In either case it follows that the orbits of $\nu^{j} z$ behave symmetrically under $z \mapsto-z$ for each $j$. Hence the Julia set of $F_{\lambda}$ is symmetric under $z \mapsto \nu z$. In particular, each of the free critical points eventually maps onto the same orbit (in case $n$ is even) or onto one of two symmetric orbits (in case $n$ is odd). Thus these orbits all have the same behavior and so the $\lambda$-plane is a natural parameter plane for each of these families.

Let $H_{\lambda}(z)$ be one of the $n$ involutions given by $H_{\lambda}(z)=\lambda^{1 / n} / z$. Then we have $F_{\lambda}\left(H_{\lambda}(z)\right)=F_{\lambda}(z)$, so that the Julia set is also preserved by each of these involutions. Note that each $H_{\lambda}$ maps the critical circle to itself and also fixes a pair of critical points $\pm \sqrt{\lambda^{1 / n}}$. Moreover, $H_{\lambda}$ maps circles centered at the origin outside the critical circle to similar circles inside the critical circle and vice versa. It follows that two such circles, one inside and one outside the critical circle, are mapped onto the same ellipse by $F_{\lambda}$.

The parameter plane (see Figures 3 and 4 ) for $F_{\lambda}$ also has several symmetries. First of all, we have

$$
\overline{F_{\lambda}(z)}=F_{\bar{\lambda}}(\bar{z})
$$

so that $F_{\lambda}$ and $F_{\bar{\lambda}}$ are conjugate via the map $z \mapsto \bar{z}$. Therefore the parameter plane is preserved by the map $\lambda \mapsto \bar{\lambda}$.

We also have $(n-1)$-fold symmetry in the parameter plane for $F_{\lambda}$. To see this, let $\omega$ be the primitive $(n-1)$ st root of unity given by $\exp (2 \pi i /(n-1))$. Then, if $n$ is even, we compute that

$$
F_{\lambda \omega}\left(\omega^{n / 2} z\right)=\omega^{n / 2}\left(F_{\lambda}(z)\right) .
$$

As a consequence, for each $\lambda \in \mathbb{C}$, the maps $F_{\lambda}$ and $F_{\lambda \omega}$ are conjugate under the linear map $z \mapsto \omega^{n / 2} z$. In particular, since, when $\lambda$ is real, the real line is preserved by $F_{\lambda}$, it follows that the straight line passing through 0 and $\omega^{n / 2}$ is preserved by $F_{\lambda \omega}$.

When $n$ is odd, the situation is a little different. We now have

$$
F_{\lambda \omega}\left(\omega^{n / 2} z\right)=-\omega^{n / 2}\left(F_{\lambda}(z)\right) .
$$


Since $F_{\lambda}(-z)=-F_{\lambda}(z)$, we therefore see that $F_{\lambda \omega}^{2}$ is conjugate to $F_{\lambda}^{2}$ via the map $z \mapsto \omega^{n / 2} z$. This means that the dynamics of $F_{\lambda}$ and $F_{\lambda \omega}$ are "essentially" the same, though subtly different. For example, if $F_{\lambda}$ has a fixed point, then under the conjugacy, this fixed point and its negative are mapped to a 2-cycle for $F_{\lambda \omega}$. Again, since the real line is invariant when $\lambda$ is real, it follows that the straight line passing through both $\pm \omega^{n / 2}$ is also invariant under $F_{\lambda \omega}$.

To summarize the symmetry properties of $F_{\lambda}$, we have:

Proposition (Symmetries in the dynamical and parameter planes). The dynamical plane for $F_{\lambda}$ is symmetric under the map $z \mapsto \nu z$ where $\nu$ is a primitive $(2 n)$ th root of unity. The parameter plane is symmetric under both $z \mapsto \bar{z}$ and $z \mapsto \omega z$ where $\omega$ is a primitive $(n-1)$ st root of unity.

The following result shows that the McMullen domain and all of the Sierpiński holes are located inside the unit circle in parameter space.

Proposition (Location of the Cantor set locus). Suppose $|\lambda| \geq 1$ and $n>2$. Then $v_{\lambda}$ lies in $B_{\lambda}$ so that $\lambda$ lies in the Cantor set locus.

Proof. Suppose $|z| \geq 2|\lambda|^{1 / 2} \geq 2$. Then we have

$$
\left|F_{\lambda}(z)\right| \geq|z|^{n}-|\lambda| /|z|^{n} \geq|z|^{n}-|\lambda|^{1-n / 2} \geq|z|^{n-1}>|z|
$$

since $n>2$. Hence $\left|F_{\lambda}^{j}(z)\right| \rightarrow \infty$ so the region $|z| \geq 2|\lambda|^{1 / 2}$ lies in $B_{\lambda}$. In particular, $v_{\lambda} \in B_{\lambda}$.

For each $n$, let $\lambda^{*}=\lambda_{n}^{*}$ be the unique real solution to the equation

$$
\left|v_{\lambda}\right|=2|\sqrt{\lambda}|=|\lambda|^{1 / 2 n}=\left|c_{\lambda}\right| \text {. }
$$

Using this equation, we compute easily that

$$
\lambda^{*}=(1 / 4)^{n /(n-1)} .
$$

The circle of radius $\lambda^{*}$ plays an important role in the parameter plane, for if $\lambda$ lies on this circle, it follows that both of the critical values lie on the critical circle for $F_{\lambda}$. If $\lambda$ lies inside this circle, then $F_{\lambda}$ maps the critical circle strictly inside itself. We call the circle of radius $\lambda^{*}$ in parameter plane the dividing circle. We will be primarily concerned in later sections with values of the parameter that lie inside the dividing circle.

2. The boundary of the basin of infinity. Our main goal in this section is to show that the boundary of $B_{\lambda}$, which we denote by $\partial B_{\lambda}$, is a simple closed curve for all parameter values that lie inside the dividing circle in parameter plane. We then derive a particular parametrization of this curve as well as several other properties of this set.

Recall that $\lambda^{*}$ is the radius of the dividing circle in the parameter plane. Let $\mathcal{O}$ be the open disk in parameter plane inside the dividing circle, so 
that $|\lambda|<\lambda^{*}$ in $\mathcal{O}$. Recall also that, if $\lambda \in \mathcal{O}$, then the critical circle $C_{\lambda}$ is mapped strictly inside itself by $F_{\lambda}$.

TheOREM. If $\lambda \in \mathcal{O}$, then the boundary of $B_{\lambda}$ is a simple closed curve.

Proof. Since the critical circle $C_{\lambda}$ is mapped strictly inside itself, there is another circle of slightly larger radius that is also mapped strictly inside itself and $n$-to-one onto an ellipse surrounding the critical segment. Call this circle $\kappa_{\lambda}$. The preimage of $\kappa_{\lambda}$ consists of a pair of simple closed curves, one of which lies strictly outside this circle. This follows since $F_{\lambda}$ maps the exterior of $C_{\lambda}$ as an $n$-to-one covering of $\mathbb{C}$ minus the critical segment. Thus this exterior preimage is mapped $n$-to-one onto $\kappa_{\lambda}$. Let $A_{\lambda}$ denote the closed annulus bounded by $\kappa_{\lambda}$ and its exterior preimage. Note that there are no critical points of $F_{\lambda}$ in $A_{\lambda}$, so $F_{\lambda}$ is an $n$-to-one covering map on all of $A_{\lambda}$.

We now quasiconformally modify $F_{\lambda}$ on $A_{\lambda}$ and its interior as follows. Let $K_{\lambda}$ be the closed disk bounded by $\kappa_{\lambda}$. We first replace $F_{\lambda}$ in $K_{\lambda}$ by a map $\widetilde{F}_{\lambda}$ that is conjugate to $z \mapsto z^{n}$ defined inside a closed disk of radius $\varrho<1$. So the new map $\widetilde{F}_{\lambda}$ takes $K_{\lambda}$ strictly inside itself. Then we further modify $F_{\lambda}$ on $A_{\lambda}-\kappa_{\lambda}$ so that the new map $\widetilde{F}_{\lambda}$ :

(i) agrees with $\widetilde{F}_{\lambda}$ on the inner boundary $\kappa_{\lambda}$ of $A_{\lambda}$;

(ii) agrees with $F_{\lambda}$ on the outer boundary of $A_{\lambda}$;

(iii) maps $A_{\lambda}-\kappa_{\lambda}$ as an $n$-to-one covering onto the annular region $K_{\lambda}-$ $\widetilde{F}_{\lambda}\left(K_{\lambda}\right)$.

Then standard arguments yield the fact that the new map $\widetilde{F}_{\lambda}$ is quasiconformally conjugate to the map $z \mapsto z^{n}$ inside the preimage of $\kappa_{\lambda}$ but agrees with $F_{\lambda}$ on the boundary of and outside this preimage. Since the preimage of $\kappa_{\lambda}$ is mapped strictly inside itself and there are no critical points (except $\infty$ ) outside this circle, the modified map is then globally conjugate to $z \mapsto z^{n}$. Hence the new map has the property that the boundary of the basin of $\infty$ is a simple closed curve. But this boundary agrees with $\partial B_{\lambda}$. Therefore $\partial B_{\lambda}$ is a quasiconformal image of the unit circle.

REMARK. Notice that this result holds for all $\lambda$-values in $\mathcal{O}$, no matter what the behavior of the critical orbit.

Because $\partial B_{\lambda}$ is a simple closed curve when $|\lambda|<\lambda^{*}$, we may invoke the theory of external rays in this case. The following facts are all well known: see [8] or [16] for details. Since there are no critical points in $B_{\lambda}$ when $\lambda \in \mathcal{O}$, there is a conjugacy $h_{\lambda}: B_{\lambda} \rightarrow \mathbb{C}-\overline{\mathbb{D}}$ between $F_{\lambda}$ on $B_{\lambda}$ and the map $z \mapsto z^{n}$ on the exterior of the unit circle in $\mathbb{C}$. This conjugacy preserves the symmetry in the dynamical plane, i.e., $h_{\lambda}(\nu z)=\nu h_{\lambda}(z)$ where $\nu$ is an $(n-1)$ st root of unity. The preimage under $h_{\lambda}$ of the straight ray $\operatorname{Arg} z=2 \pi \theta$ is called the external ray of angle $\theta$. Note that these angles are measured mod 1 . Since 
$\partial B_{\lambda}$ is a simple closed curve, it is known that each external ray limits on a unique point in the boundary of $B_{\lambda}$ called the landing point of the external ray. We denote the landing point of the external ray of angle $\theta$ by $z_{\lambda}(\theta)$. Then the conjugacy $h_{\lambda}$ extends to $\partial B_{\lambda}$ and we have $F_{\lambda}\left(z_{\lambda}(\theta)\right)=z_{\lambda}(n \theta)$. Moreover, if $\theta$ is periodic of period $k$ under the map $\theta \mapsto n \theta \bmod 1$, then the landing point $z_{\lambda}(\theta)$ is also periodic of period $k$ for $F_{\lambda}$. Hence such periodic points are dense in $\partial B_{\lambda}$. Now each periodic point of the form $z_{\lambda}(\theta)$ varies analytically with $\lambda$. Since these points are dense in $\partial B_{\lambda}$, it follows that the map $\lambda \mapsto z_{\lambda}(\theta)$ is a holomorphic motion, and so $z_{\lambda}(\theta)$ varies analytically in $\lambda$ for all values of $\theta$, not just those that correspond to periodic points. Also, $z_{\lambda}(\theta)$ varies continuously with $\theta$. The function $\theta \mapsto z_{\lambda}(\theta)$ is thus the parametrization of $\partial B_{\lambda}$.

We can now use the involutions $H_{\lambda}$ to construct a similar parametrization of the $\partial T_{\lambda}$. Fix any $n$th root of $\lambda$ and let $H_{\lambda}$ be the corresponding involution $\lambda^{1 / n} / z$. Then define $w_{\lambda}(\theta)=H_{\lambda}\left(z_{\lambda}(\theta)\right)$. Since $H_{\lambda}$ interchanges the boundaries of $B_{\lambda}$ and $T_{\lambda}$, the function $\theta \mapsto w_{\lambda}(\theta)$ parametrizes the boundary of $T_{\lambda}$. We get different parametrizations of $\partial T_{\lambda}$ depending upon which root of $\lambda$ we choose.

The following result gives a bound on the location of the boundary of $B_{\lambda}$ as well as a criterion for when a point belongs to $B_{\lambda}$.

Proposition (The escape criterion). Suppose $|\lambda| \leq \lambda^{*}$. Then the boundary of $B_{\lambda}$ is strictly contained in an open annulus that lies between $C_{\lambda}$ and the circle of radius 1.2 centered at the origin. The inner (resp., outer) boundary of this annulus is mapped strictly inside (resp., outside) itself by $F_{\lambda}$. Moreover, if $|z| \geq 1.2$, then $z \in B_{\lambda}$. If $|z| \leq|\lambda|^{1 / n} / 1.2$, then $z \in T_{\lambda}$ and $F_{\lambda}(z) \in B_{\lambda}$.

Proof. If $|z| \geq 1.2$ and $|\lambda| \leq \lambda^{*}=(1 / 4)^{n /(n-1)}<1 / 4$, we have

$$
\left|F_{\lambda}(z)\right| \geq|z|^{n}-\frac{|\lambda|}{|z|^{n}} \geq|z|^{n}-\frac{1}{4(1.2)^{n}}>|z|^{n}-0.15>|z|^{2}>|z| .
$$

It follows that $F_{\lambda}$ maps each circle of the form $|z|=r \geq 1.2$ strictly outside itself and hence the entire region $|z| \geq 1.2$ is contained in $B_{\lambda}$.

If $|\lambda|<\lambda^{*}$, the critical circle of $F_{\lambda}$ is mapped strictly inside itself and so the boundary of $B_{\lambda}$ lies outside $C_{\lambda}$. Therefore the boundary of $B_{\lambda}$ must lie somewhere strictly inside the annulus bounded by $C_{\lambda}$ and circle of radius 1.2 centered at the origin if $|\lambda|<\lambda^{*}$. If $|\lambda|=\lambda^{*}$, we need a slightly different argument. In this case the critical segment now meets $C_{\lambda}$ in exactly two points, namely $\pm v_{\lambda}$. Consider the preimage of $C_{\lambda}$ that lies on or outside $C_{\lambda}$; call this preimage $D_{\lambda}$. This preimage consists of exactly $2 n$ smooth arcs having the properties:

(i) each arc meets $C_{\lambda}$ in exactly two consecutive critical points; 
(ii) all non-critical points on $D_{\lambda}$ lie outside $C_{\lambda}$;

(iii) each arc in $D_{\lambda}$ is mapped onto exactly one-half of the critical circle connecting $+v_{\lambda}$ to $-v_{\lambda}$ in one direction or the other.

As a consequence, if $\pm v_{\lambda}$ are not themselves critical points, then $D_{\lambda}$ is a simple closed curve that is mapped strictly inside itself, and so the boundary of $B_{\lambda}$ again lies outside of $D_{\lambda}$ and hence outside of $C_{\lambda}$.

If the points $\pm v_{\lambda}$ are also critical points, then it follows that we have one of three cases: $\pm v_{\lambda}$ are both superattracting fixed points, one is superattracting and fixed while the other is pre-fixed, or else they lie on a superattracting 2-cycle. In each of these cases, neighborhoods of $\pm v_{\lambda}$ are contracted inside themselves, and so we can modify $D_{\lambda}$ to a slightly different curve near $\pm v_{\lambda}$ which is again mapped strictly inside itself. This produces the inner boundary of the annulus containing $\partial B_{\lambda}$.

Finally, recall that the involution $H(z)=\lambda^{1 / n} / z$ has the property that $F_{\lambda}(H(z))=F_{\lambda}(z)$. This involution takes the region $|z| \geq 1.2$ to the closed disk of radius $|\lambda|^{1 / n} / 1.2$ about the origin. Hence any point in this disk is mapped into $B_{\lambda}$ and so this disk lies in the trap door.

Corollary. If $|\lambda| \leq \lambda^{*}$, then neither $B_{\lambda}$ nor the boundary of $B_{\lambda}$ meets the critical circle. Similarly, neither $T_{\lambda}$ nor the boundary of $T_{\lambda}$ meets the critical circle.

3. Extended rays. We say that a region $\mathcal{R}$ is an open sector of width $\delta$ if

$$
\mathcal{R}=\{z \mid \alpha<\operatorname{Arg} z<\alpha+\delta\}
$$

for some $\alpha$. A closed sector of width $\delta$ is defined analogously. In order to construct a parametrization of the boundary of the McMullen domain, we need the following result that gives a bound on the location of points in the parametrization of $\partial T_{\lambda}$.

Proposition. Fix an angle $\theta$. Then there exists an open sector $\mathcal{R}$ of width $3 \pi / n$ such that $z_{\lambda}(\theta)$ is contained in a compact subset of $\mathcal{R}$ for all $\lambda \in \overline{\mathcal{O}}$.

Before turning to the technical proof of this result, we first introduce the notion of an extended ray. A simple closed curve $\zeta_{0}$ is called an extended ray of angle 0 if it has the following properties:

(i) $\zeta_{0}$ passes through the origin and $\infty$ and is invariant under $z \mapsto-z$;

(ii) $F_{\lambda}$ maps $\zeta_{0}$ to itself in two-to-one fashion;

(iii) $\zeta_{0}$ contains the entire external rays of angles 0 and $1 / 2$;

(iv) there is a compact subset of $\zeta_{0}$ homeomorphic to a Cantor set and on which $F_{\lambda}$ is conjugate to the 2-shift; 
(v) all points in $\zeta_{0}$ that do not lie in this Cantor set have orbits that eventually land on the external rays of angle 0 or $1 / 2$.

For $j \in \mathbb{Z}$, let $\zeta_{j}=\nu^{j} \cdot \zeta_{0}$ where $\nu=\exp (\pi i / n)$. Then, using the symmetry properties of $F_{\lambda}$, we have $F_{\lambda}\left(\zeta_{j}\right)=\zeta_{0}$. We call $\zeta_{j}$ the extended ray of angle $j / 2 n$. We see that $\zeta_{j}=\zeta_{j+n}$ for each $j$ since $\zeta_{j}$ contains the external rays of angles $j / 2 n$ and $(j+n) / 2 n$.

To construct $\zeta_{0}$, we first assume that $\lambda \notin \mathbb{R}^{+}$. Then we index the $2 n$ critical points of $F_{\lambda}$ by $c_{j}=c_{j}(\lambda), j=0, \ldots, 2 n-1$, as follows. Since $\lambda \notin \mathbb{R}^{+}$, there is a unique critical point $c_{0}$ that satisfies

$$
0<\operatorname{Arg} c_{0}<\pi / n .
$$

We index the remaining critical points in increasing order in the counterclockwise direction around the critical circle.

Now consider the closed prepole sector $\mathcal{P}_{\lambda}^{+}=\mathcal{P}^{+}$of angle $\pi / n$ bounded by the two straight lines connecting the origin to $\infty$ and passing through the critical points $c_{0}$ and $c_{2 n-1}$. Let $\mathcal{P}_{\lambda}^{-}=\mathcal{P}^{-}=-\mathcal{P}^{+}$and set

$$
\mathcal{P}_{\lambda}^{\prime}=\mathcal{P}^{\prime}=\mathcal{P}^{+} \cup \mathcal{P}^{-} \cup\{\infty\}
$$

Proposition. Let $\zeta_{0}=\zeta_{0}(\lambda)$ be the set of points whose orbits remain in $\mathcal{P}^{\prime}$ for all iterations. Then $\zeta_{0}$ is an extended ray of angle 0.

Proof. Since $\lambda \notin \mathbb{R}^{+}$, the straight line boundaries of $\mathcal{P}^{\prime}$ are mapped strictly outside $\mathcal{P}^{\prime}$, except at 0 and $\infty$. Indeed, the images of these straight rays are the straight rays connecting $\pm v_{\lambda}$ to $\infty$, and these image rays never meet $\mathcal{P}$ since $\pm v_{\lambda} \notin \mathcal{P}^{\prime}$. In particular, $F_{\lambda}$ maps the interior of both $\mathcal{P}^{+}$and $\mathcal{P}^{-}$one-to-one onto the entire plane minus these two rays.

Let $\mathcal{Q}^{+}$be the closed subset of $\mathcal{P}^{+}$lying on or outside the critical circle. Since the portion of the critical circle bounding $\mathcal{Q}^{+}$is mapped to the critical segment, it follows that $F_{\lambda}$ also maps $\mathcal{Q}^{+}$strictly outside itself (except at $\infty$ ). Hence there exists a unique fixed external ray lying in $\mathcal{Q}^{+}$. Without loss of generality, we take this to be the external ray of angle $\theta=0$. Moreover, there exists $r \gg 1$ such that the circle of radius $r$ is mapped strictly outside itself in $B_{\lambda}$. If we then restrict attention to the portion of $\mathcal{Q}^{+}$that lies inside this circle, it follows that $F_{\lambda}$ maps this simply connected region strictly over itself. Hence there exists a unique repelling fixed point in this region. This then is the fixed point $z_{\lambda}(0)$ lying in $\partial B_{\lambda}$ and serving as the landing point of the external ray of angle 0 . So $\zeta_{0}$ contains at least the external ray of angle 0 and its landing point, since the orbits of these points never leave $\mathcal{P}^{+}$. Note that any point in $\mathcal{Q}^{+}$(with the exception of those on the external ray of angle 0 and $\left.z_{\lambda}(0)\right)$ has orbit that leaves $\mathcal{Q}^{+}$.

By the $z \mapsto-z$ symmetry, the region $\mathcal{P}^{-}$also contains the external ray of angle $1 / 2$ together with $z_{\lambda}(1 / 2)$. The point $z_{\lambda}(1 / 2)$ and its external ray 
may be fixed or pre-fixed; this depends on whether $n$ is odd or even. In any event, this ray and its landing point also lie in $\zeta_{0}$.

Since $F_{\lambda}$ maps $\mathcal{P}^{+}$one-to-one over all of $\mathcal{P}^{\prime}$, there is a curve lying in $\mathcal{P}^{+}$ and terminating at the origin that is mapped to the external ray of angle $1 / 2$ and so this curve also lies in $\zeta_{0}$. By symmetry there is a similar curve in $\mathcal{P}^{-}$.

Thus far, $\zeta_{0}$ consists of two curves in $\mathcal{P}^{\prime}$ : one passing through $\infty$ and containing the two external rays, and another curve passing through the origin which is mapped onto the curve containing the external rays. Our goal is to extend this curve by taking further preimages of $F_{\lambda}$ in $\mathcal{P}^{\prime}$.

First, however, we note that there is an invariant Cantor set lying in $\mathcal{P}$. Indeed, let $\mathcal{R}$ denote the portion of $\mathcal{P}^{\prime}$ lying inside the circle of radius $r$ constructed above, and outside the image of this circle under any of the involutions $H_{\lambda}$. So $\mathcal{R}$ is the intersection of the sectors $\mathcal{P}^{ \pm}$with an annulus. Let $\mathcal{R}^{ \pm}=\mathcal{R} \cap \mathcal{P}^{ \pm}$. Then $F_{\lambda}$ maps each of $\mathcal{R}^{ \pm}$one-to-one over both of these sets, with the boundaries of each mapped strictly outside of $\mathcal{R}^{ \pm}$. Standard arguments then show that the set of points whose orbits remain for all time in $\mathcal{R}$ is a Cantor set, and the restriction of $F_{\lambda}$ to this Cantor set is conjugate to the shift map on two symbols. Since the orbits of any point in this Cantor set lie for all iterations in $\mathcal{R} \subset \mathcal{P}$, it follows that the entire Cantor set also lies in $\zeta_{0}$. Note that the points $z_{\lambda}(0)$ and $z_{\lambda}(1 / 2)$ are, by construction, part of this Cantor set, as are their two preimages in $\mathcal{P}^{\prime}$. We think of these points as being "endpoints" of the Cantor set. Note also that any point in $\zeta_{0}$ that lies in $\mathcal{R}$ but not in the Cantor set must have orbit that leaves $\mathcal{R}$. Hence this orbit must eventually land on one of the two external rays lying in $\zeta_{0}$.

We may thus pull back these two external rays lying in $\zeta_{0}$ using appropriate branches of the inverse of $F_{\lambda}$ taking values in $\mathcal{P}^{ \pm}$. Each pullback gives a collection of curves connecting various preimages of the endpoints of the Cantor set. And the union of these preimages provides all of the remaining points in $\zeta_{0}$. Since the action of $F_{\lambda}$ on the Cantor set is the 2-shift, we can then construct a homeomorphism between $\zeta_{0}$ and the real line that takes:

(i) the Cantor set portion of $\zeta_{0}$ to the Cantor middle-thirds set in the unit interval;

(ii) the external rays of angle 0 and $1 / 2$ to the intervals $(1, \infty)$ and $(-\infty, 0)$ respectively;

(iii) the various preimages of these rays to the appropriate middle-thirds removed intervals.

This then yields the curve $\zeta_{0}$. Note that, by construction, $F_{\lambda}$ is two-to-one on $\zeta_{0}$.

REMARK. The extended ray $\zeta_{0}$ and its various preimages $\zeta_{j}$ together with the boundaries of $B_{\lambda}$ and $T_{\lambda}$ may be used to describe the symbolic 
dynamics associated with the action of $F_{\lambda}$ on the Julia set. See [6] for more details concerning this construction.

Corollary. For each $\lambda \in \overline{\mathcal{O}}-\left[0, \lambda^{*}\right]$, the extended ray $\zeta_{0}$ lies in the sector $|\operatorname{Arg} z|<\pi / n$.

Proof. This follows from the fact that, since $\lambda \notin \mathbb{R}^{+}$, the critical points $c_{0}$ and $c_{2 n-1}$ satisfy

$$
0<\operatorname{Arg} c_{0}<\pi / n \text { and }-\pi / n<\operatorname{Arg} c_{2 n-1}<0 .
$$

The rays containing $c_{0}$ and $c_{2 n-1}$ make up the boundary rays of $\mathcal{P}_{\lambda}^{+}$for each $\lambda$.

Lemma. The only two points in the Cantor set portion of $\zeta_{0}$ whose entire orbits lie on or outside of the critical circle are the fixed point $z_{\lambda}(0)$ in $\mathcal{P}^{+}$ and $-z_{\lambda}(0)$ in $\mathcal{P}^{-}$.

Proof. $F_{\lambda}$ maps the portion of $\mathcal{P}^{+}$that lies on or outside of the critical circle to a half-plane that covers $\mathcal{P}^{+}$and misses $\mathcal{P}^{-}$. Hence the only orbit that stays for all time in $\mathcal{P}^{+}$and does not tend to $\infty$ is the fixed point $z_{\lambda}(0)$. By symmetry, $F_{\lambda}$ maps the portion of $\mathcal{P}^{-}$outside the critical circle to the same half-plane (if $n$ is even) or to the symmetric half-plane (if $n$ is odd). In either case, only $-z_{\lambda}(0)$ has orbit that stays in the portions of these two sectors outside the critical circle and does not tend to $\infty$.

We now turn to the proof of the proposition that says that, for any $\lambda \in \overline{\mathcal{O}}$, the point $z_{\lambda}(\theta)$ always lies in a compact subset of an open sector of width $3 \pi / n$ that is bounded away from the origin. We first recall that the function $\lambda \mapsto z_{\lambda}(\theta)$ depends analytically on $\lambda$. Hence the image of $\overline{\mathcal{O}}$ under this function is always a compact set. Thus we must show that each such point lies in an open sector of the appropriate size. Secondly, by the dynamical plane symmetry, it suffices to consider only $\theta$-values in the interval $[0,1 / 2 n]$. We shall show that, for any such $\theta$, the point $z_{\lambda}(\theta)$ always lies in the open sector $\mathcal{S}$ bounded by the rays $\operatorname{Arg} z=-\pi / n$ and $\operatorname{Arg} z=2 \pi / n$.

Under the assumption that $\lambda \notin \mathbb{R}^{+}$, we know that the extended ray $\zeta_{0}(\lambda)$ (excluding the origin and $\infty$ ) lies in a pair of symmetric open sectors of width $\pi / n$ that in turn are always contained in the union of the open sector $\operatorname{Arg} z= \pm \pi / n$ and its negative. By the $2 n$-fold symmetry of $F_{\lambda}$, the extended ray $\zeta_{1}(\lambda)=\nu \cdot \zeta_{0}(\lambda)$ contains the external rays of angles $1 / 2 n$ and $(n+1) / 2 n$. Note that $F_{\lambda}$ takes $\zeta_{1}$ two-to-one onto $\zeta_{0}$ and that $\zeta_{1}$ (again excluding the origin and $\infty$ ) always lies in the open sector given by

$$
0<\operatorname{Arg} z<2 \pi / n
$$

and its negative. Since the external rays of angles 0 and $1 / 2 n$ and their landing points always lie along the portions of $\zeta_{0}$ and $\zeta_{1}$ in $\mathcal{S}$, this proves the result in the special cases where $\theta=0$ or $\theta=1 / 2 n$. 
So now consider an external ray $\gamma$ of angle $\theta$ with $\theta \in(0,1 / 2 n)$. This external ray cannot meet any point on either $\zeta_{0}(\lambda)$ or $\zeta_{1}(\lambda)$ that eventually maps to the external rays of angles 0 or $1 / 2 n$ since external rays do not intersect. We claim that this ray also cannot meet any point in the Cantor set portion of these two extended rays. Certainly this ray does not meet $\pm z_{\lambda}(0)$ since these are the landing points of the external rays of angles 0 and $1 / 2$, and no two rays land at the same point. Also, if this ray were to land at some other point in the Cantor set, then, by the Lemma, some point on the orbit of this point must lie inside the critical circle. Hence some iterate of this ray must meet the critical circle. But this is impossible, since $B_{\lambda}$ and all of the external rays lie strictly outside the critical circle by the Corollary at the end of the previous section.

Thus it follows that $\gamma$ is trapped between the two portions of the curves $\zeta_{0}$ and $\zeta_{1}$ that lie in the right half-plane. But these curves lie in the open sector bounded by $\operatorname{Arg} z=-\pi / n$ and $\operatorname{Arg} z=2 \pi / n$. Hence the landing point of this ray is also trapped in this region. This proves the result as long as $\lambda \notin \mathbb{R}^{+}$.

In the special case where $\lambda \in \mathbb{R}^{+}$, there is an ambiguity in the definition of the sectors $\mathcal{P}^{ \pm}$, since, as $\lambda$ rotates once around the origin, these sectors each rotate by $\pi / n$ radians. However, there is no ambiguity in the definition of the external ray of angles $\theta$ nor its landing point $z_{\lambda}(\theta)$. This follows since, when $\lambda \in \mathbb{R}^{+}$, the real line is invariant, and the external rays of angles 0 and $1 / 2$ both lie in $\mathbb{R}$. Similarly, the rays of arguments $j \pi / n$ are all mapped to $\mathbb{R}$. Hence these lines each contain the external rays of angle $j \pi / n$ and so no other external ray can intersect these lines as shown above. This takes care of the case $\lambda \in \mathbb{R}^{+}$.

Finally, we note that $z_{\lambda}(\theta)$ lies in a compact portion of $\mathcal{S}$ bounded away from the origin. Indeed, for any $\lambda \in \mathcal{O}$, by the escape criterion, $\partial B_{\lambda}$ is contained inside the circle of radius 2 centered at the origin and outside the trap door. Since the boundary of $T_{\lambda}$ lies outside of the circle of radius $|\lambda|^{1 / n} / 2$, this proves the result provided $\lambda \neq 0$. But if $\lambda=0$, then all points of the form $z_{0}(\theta)$ lie on the unit circle and so the set containing all such $z_{\lambda}(\theta)$ is a compact subset of $\mathcal{S}$ bounded away from the origin. This completes the proof.

4. The McMullen domain. Let $\mathcal{M}$ denote the McMullen domain, i.e.

$$
\mathcal{M}=\left\{\lambda \mid v_{\lambda} \in T_{\lambda}\right\},
$$

so that the Julia set of $F_{\lambda}$ is a Cantor set of circles if $\lambda \in \mathcal{M}$. In this section we combine the results of the previous two sections to show that, for each $n \geq 3, \mathcal{M}$ consists of a single, open, simply connected region surrounding the origin and bounded by a simple closed curve. 
We first show that there is a component of the McMullen domain surrounding the origin in the parameter plane.

Proposition. Suppose that

$$
|\lambda|<(1 / 4)^{2 n /(n-2)} .
$$

Then $v_{\lambda}$ lies in $T_{\lambda}$ so $\lambda \in \mathcal{M}$.

Proof. Since $n \geq 3$, we have

$$
\frac{2 n}{n-2}>\frac{n}{n-1}
$$

Therefore

$$
|\lambda|<(1 / 4)^{2 n /(n-2)}<(1 / 4)^{n /(n-1)}=\lambda^{*} .
$$

Now

$$
|\lambda|^{1 / 2-1 / n}=|\lambda|^{(n-2) / 2 n}<1 / 4
$$

so we have

$$
\left|v_{\lambda}\right|=2|\lambda|^{1 / 2}<\frac{|\lambda|^{1 / n}}{2}<\frac{|\lambda|^{1 / n}}{1.2} .
$$

By the escape criterion in Section 2 , it follows that $v_{\lambda} \in T_{\lambda}$. So $\lambda \in \mathcal{M}$.

This Proposition proves the existence of a component of the McMullen domain that surrounds the origin in parameter space when $n \geq 3$. We denote this component by $\mathcal{M}_{0}$; we next show that $\mathcal{M}_{0}$ is bounded by a simple closed curve.

Recall that, for each $\lambda \in \mathcal{O}$, the boundary of $B_{\lambda}$ is a simple closed curve that can be parametrized by the function $\theta \mapsto z_{\lambda}(\theta)$. Similarly, the boundary of $T_{\lambda}$ is a simple closed curve that we can parametrize by the function $\theta \mapsto w_{\lambda}(\theta)$. Moreover, the functions $z_{\lambda}(\theta)$ and $w_{\lambda}(\theta)$ depend analytically on $\lambda$.

TheOREM. For each angle $\theta$, there is a unique $\lambda=\lambda_{\theta} \in \mathcal{O}$ such that $v_{\lambda_{\theta}}=w_{\lambda_{\theta}}(\theta)$. In particular, $\lambda_{\theta}$ lies on the boundary of $\mathcal{M}_{0}$ and $\lambda \mapsto \lambda_{\theta}$ parametrizes this boundary.

Proof. Fix an angle $\theta$. From the results in Section 3, we know that for each $\lambda \in \mathcal{O}$, the point $z_{\lambda}(\theta)$ lies in a compact subset of a sector of the form

$$
\alpha<\operatorname{Arg} z<\alpha+3 \pi / n
$$

where $\alpha$ is independent of $\lambda$. Furthermore, by the escape criterion, each $z_{\lambda}(\theta)$ lies outside $C_{\lambda}$ in this sector. Note that, for different values of $\theta$, we must choose different values of $\alpha$. As shown in the construction of the extended rays, for $\theta \in[0,1 / 2 n]$, we may choose $\alpha=-\pi / n$. But for larger values of $\theta$, we must rotate this sector. In particular, as $\theta$ winds once around the circle, the sectors determined by $\alpha$ also wind once around the origin. 
If we apply any of the involutions $H_{\lambda}$ to this sector, we obtain another similar sector, say $\mathcal{S}$, which is of the form

$$
\beta<\operatorname{Arg} z<\beta+3 \pi / n
$$

where now $\beta$ is independent of $\lambda$. Moreover, for each $\lambda \in \mathcal{O}, w_{\lambda}(\theta)$ lies in a compact portion of $\mathcal{S}$ that is bounded away from the origin but inside $C_{\lambda}$. For $\lambda \in \mathcal{O}$, the largest radius that the critical circle can have occurs when $|\lambda|=\left(\lambda^{*}\right)^{1 / 2 n}$.

When $n=3$, the sector $\mathcal{S}$ is a half-plane and, when $n>3, \mathcal{S}$ is smaller than a half-plane. Let $\ell$ be the ray in parameter plane that consists of all $\lambda$-values whose argument is of the form $-(\beta+3 \pi / 2 n)$. So $\ell$ is the negative of the ray that is the central line in $\mathcal{S}$ and so $\ell$ lies outside $\mathcal{S}$. Moreover, if $\lambda \in \mathbb{C}-\ell$, then we may choose a branch of the square root of $\lambda$ that maps $\mathbb{C}-\ell$ onto a half-plane that contains $\mathcal{S}$. Let $G(\lambda)=2 \sqrt{\lambda}$ denote this branch.

Let $\mathcal{O}^{\prime}=\mathcal{O}-\ell$ and consider $G \mid \mathcal{O}^{\prime}$. The largest magnitude that $G$ can achieve in $\mathcal{O}^{\prime}$ is $2 \sqrt{\lambda^{*}}$, but recall that $2 \sqrt{\lambda^{*}}=\left(\lambda^{*}\right)^{1 / 2 n}$. Hence $G$ maps $\mathcal{O}^{\prime}$ one-to-one onto the portion of the disk of this radius in the half-plane that contains $\mathcal{S}$. Therefore $G\left(\mathcal{O}^{\prime}\right)$ covers the compact region that contains each $w_{\lambda}(\theta)$ for each $\theta$. So, for each fixed value of $\theta$, we may consider the function $P_{\theta}: \mathcal{O}^{\prime} \rightarrow \mathcal{O}^{\prime}$ defined by

$$
P_{\theta}(\lambda)=G^{-1}\left(w_{\lambda}(\theta)\right) .
$$

$P_{\theta}$ is analytic in $\lambda$ and maps the simply connected region $\mathcal{O}^{\prime}$ to a compact subset of itself. By the Schwarz Lemma, there exists a unique fixed point $\lambda_{\theta}$ for this map. For the parameter value $\lambda_{\theta}$, the critical value $2 \sqrt{\lambda_{\theta}}$ therefore lies at the point $w_{\lambda_{\theta}}(\theta) \in \partial T_{\lambda_{\theta}}$.

We claim that $\lambda_{\theta}$ lies on the boundary of $\mathcal{M}$. To prove this we must show that there are $\lambda$-values arbitrarily close to $\lambda_{\theta}$ for which $v_{\lambda} \in T_{\lambda}$. For $\lambda \in \mathcal{O}$ and $r \geq 1$, let $\gamma_{\lambda}(r)$ denote the point on the external ray of the given angle $\theta$ for $F_{\lambda}$ for which $\left|h_{\lambda}\left(\gamma_{\lambda}(r)\right)\right|=r$, i.e., $\gamma_{\lambda}(r)$ is the unique point on this external ray that is mapped to the circle of radius $r$ via the conjugacy $h_{\lambda}$. So $\gamma_{\lambda}(1)=z_{\lambda}(\theta)$ is the landing point in $\partial B_{\lambda}$ of the external ray of angle $\theta$.

It is known that the function $\lambda \mapsto \gamma_{\lambda}(r)$ varies analytically in $\lambda$ and continuously in $r$. For $r$ close to 1, we may thus consider the analytic function $\lambda \mapsto G^{-1}\left(H_{\lambda}\left(\gamma_{\lambda}(r)\right)\right)$. For $r$ sufficiently close to $1, \gamma_{\lambda}(r)$ also lies in a compact subset of $\mathcal{O}^{\prime}$ in $\mathcal{S}$. As above, there then exists a unique fixed point $\lambda_{r}$ for this function. But this means that $F_{\lambda_{r}}\left(v_{\lambda_{r}}\right) \in B_{\lambda_{r}}$. However, $v_{\lambda_{r}} \notin B_{\lambda_{r}}$ since $\lambda_{r} \in \mathcal{O}$ so that $B_{\lambda_{r}}$ lies outside the critical circle whereas $v_{\lambda_{r}}$ lies inside this circle. Thus $\lambda_{r} \in T_{\lambda_{r}}$. This shows that there are $\lambda$-values in any neighborhood of $\lambda_{\theta}$ that also lie in $\mathcal{M}$, so $\lambda_{\theta} \in \partial \mathcal{M}$.

Now the parameter $\lambda_{\theta}$ depends continuously on $\theta$. Thus the function $\theta \mapsto \lambda_{\theta}$ parametrizes a simple closed curve in $\mathcal{O}$ which therefore serves as 
the boundary of a single component of $\mathcal{M}$. Since the curve $\lambda_{\theta}$ surrounds the origin in parameter space, this component must therefore be $\mathcal{M}_{0}$.

As a consequence, we have the fact that $\mathcal{M}_{0}$ is an open and simply connected subset of parameter plane. We finally must show that $\mathcal{M}_{0}$ is the only component of the McMullen domain. Suppose $\mathcal{N}$ is a second component of $\mathcal{M}$. Then $\mathcal{N}$ cannot meet the dividing circle in the parameter plane. This follows since, if $|\lambda|=\lambda^{*}$, then we know that $\bar{T}_{\lambda}$ lies strictly inside the critical circle whereas $v_{\lambda}$ lies on the critical circle in this case. So we cannot have $v_{\lambda} \in T_{\lambda}$. Also, using the following result, we deduce that, in the complement of $\mathcal{M}_{0}$ and the Cantor set locus in $\mathbb{R}^{+}$, the orbit of the critical point in $\mathbb{R}^{+}$ is bounded. Hence $\mathcal{N}$ cannot meet $\mathbb{R}^{+}$:

THEOREM. There is a homeomorphic copy of the Mandelbrot set lying symmetrically about $\mathbb{R}^{+}$in parameter plane. The cusp of the main cardioid in this set lies on the boundary of the connectedness locus, while the Misiurewicz parameter at the tip of the tail of this set lies on the boundary of $\mathcal{M}_{0}$.

For a proof, we refer to [3]. By the parameter plane symmetry, it also follows that $\mathcal{N}$ cannot meet any of the lines $t \omega$ where $t>0$ and $\omega$ is an $(n-1)$ st root of unity, for there we have a similar copy of a Mandelbrot set.

We next claim that $\mathcal{N}$ is simply connected. To see this, let $\gamma$ be a simple closed curve in $\mathcal{N}$. Then $\gamma$ cannot surround the origin, since $\mathcal{N}$ is confined to lie in a sector bounded by $\omega^{j} \cdot \mathbb{R}^{+}$and $\omega^{j+1} \cdot \mathbb{R}^{+}$for some $j$. Hence we can choose a well defined branch of $v_{\lambda}=2 \sqrt{\lambda}$ on and in the interior of $\gamma$. Since $\gamma$ is compact and lies in $\mathcal{N}$, there exists an $n$ such that, for each $\lambda \in \gamma$, $F_{\lambda}^{n}\left(v_{\lambda}\right)$ lies outside the circle of radius 2 and hence $F_{\lambda}^{n}\left(v_{\lambda}\right) \in B_{\lambda}$. Since the function $N_{n}(\lambda)=F_{\lambda}^{n}\left(v_{\lambda}\right)$ is analytic, it follows that all $\lambda$-values inside $\gamma$ are also mapped outside the circle of radius 2 . But then these $\lambda$-values must all lie in $\mathcal{N}$ since they all escape to $\infty$ just as those on $\gamma$ do. Hence $\mathcal{N}$ is simply connected.

Now consider the function $N_{n}(\lambda)=F_{\lambda}^{n}\left(v_{\lambda}\right)$ defined on all of $\overline{\mathcal{N}}$. This function never takes the value $\infty$ since $v_{\lambda}=2 \sqrt{\lambda} \neq 0$ for any $\lambda \in \overline{\mathcal{N}}$. If $\lambda \in \partial \mathcal{N}$, then $\left|N_{n}(\lambda)\right|$ is bounded by 2 for all $n$ since the orbit of $v_{\lambda}$ does not escape to $\infty$. But $N_{n}$ takes arbitrarily large values for $n$ large and $\lambda \in \mathcal{N}$. This means that $N_{n}$ violates the Maximum Principle and so $N_{n}$ is not analytic. This yields a contradiction and shows that no such domain $\mathcal{N}$ exists. This proves that $\mathcal{M}_{0}$ is the unique component of $\mathcal{M}$.

\section{References}

[1] P. Blanchard, R. L. Devaney, D. M. Look, P. Seal and Y. Shapiro, Sierpiński curve Julia sets and singular perturbations of complex polynomials, Ergodic Theory Dynam. Systems, to appear. 
[2] R. L. Devaney, Cantor and Sierpiński, Julia and Fatou: complex topology meets complex dynamics, Notices Amer. Math. Soc. 51 (2004), 9-15.

[3] —, Baby Mandelbrot sets adorned with halos, to appear.

[4] R. L. Devaney, K. Josić and Y. Shapiro, Singular perturbations of quadratic maps, Internat. J. Bifur. Chaos Appl. Sci. Engrg. 14 (2004), 161-169.

[5] R. L. Devaney and D. M. Look, A criterion for Sierpinski curve Julia sets, to appear.

[6] —, - Symbolic dynamics for a Sierpiński curve Julia set, Internat. J. Differential Equations Appl., to appear.

[7] R. L. Devaney, D. M. Look and D. Uminsky, The escape trichotomy for singularly perturbed rational maps, Indiana Univ. Math. J., to appear.

[8] A. Douady et J. H. Hubbard, Étude dynamique des polynômes complexes, Publ. Math. d'Orsay 84-2, Univ. Paris-Sud, Orsay, 1984.

[9] - - - On the dynamics of polynomial-like mappings, Ann. Sci. École Norm. Sup. (4) 18 (1985), 287-343.

[10] R. Mañé, P. Sad and D. Sullivan, On the dynamics of rational maps, ibid. 16 (1983), 193-217.

[11] C. McMullen, Automorphisms of rational maps, in: Holomorphic Functions and Moduli, Vol. 1, Math. Sci. Res. Inst. Publ. 10, Springer, New York, 1988, 31-60.

[12] - The classification of conformal dynamical systems, in: Current Developments in Mathematics, Internat. Press, Cambridge, MA, 1995, 323-360.

[13] J. Milnor, Dynamics in One Complex Variable, Vieweg, Braunschweig, 1999.

[14] J. Milnor and L. Tan, A Sierpiński carpet as Julia set, Appendix F in: Geometry and dynamics of quadratic rational maps, Experiment. Math. 2 (1993), 37-83.

[15] S. Nadler, Continuum Theory: An Introduction, Marcel Dekker, New York, 1992.

[16] C. Petersen and G. Ryd, Convergence of rational rays in parameter spaces, in: The Mandelbrot Set: Theme and Variations, London Math. Soc. Lecture Note Ser. 274, Cambridge Univ. Press, Cambridge, 2000, 161-172.

[17] P. Roesch, On captures for the family $f_{\lambda}(z)=z^{2}+\lambda / z^{2}$, to appear.

[18] S. Ushiki, Julia sets with polyhedral symmetries, in: Fractals in the Fundamental and Applied Sciences, H.-O., Peitgen, J. M. Henriques and L. F. Penedo (eds.), Elsevier, 1991, 425-442.

[19] G. T. Whyburn, Topological characterization of the Sierpinski curve, Fund. Math. 45 (1958), 320-324.

Boston University

Boston, MA 02215, U.S.A.

E-mail: bob@bu.edu

Received 11 January 2005;

in revised form 25 March 2005 